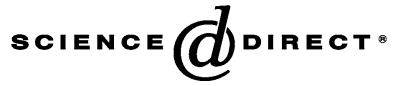

Gynecologic Oncology 97 (2005) 529-534
Gynecologic Oncology

www.elsevier.com/locate/ygyno

\title{
CA-125 AUC as a new prognostic factor for patients with ovarian cancer
}

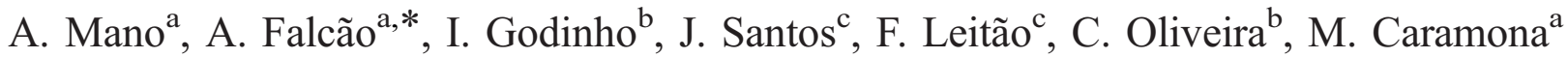 \\ ${ }^{a}$ Laboratory of Pharmacology, Faculty of Pharmacy, University of Coimbra, Portugal \\ ${ }^{\mathrm{b}}$ Gynaecology Service, Coimbra University Hospital, 3000-295 Coimbra, Portugal \\ cPathology Service, Coimbra University Hospital, 3000-295 Coimbra, Portugal
}

Received 14 November 2004

Available online 17 March 2005

\begin{abstract}
Objective. The aim of the present study was to investigate the usefulness of the CA-125 area under the curve (AUC) as a new kinetic parameter for predicting overall survival in patients with ovarian cancer. In addition, the relationship of CA-125 AUC with other prognostic factors of ovarian cancer was evaluated.

Methods. Ninety-two patients that underwent primary line chemotherapy within 4 months after submission to cytoreductive surgery were included. For each patient, CA-125 AUC was calculated and a statistical analysis was conducted to compare CA-125 AUC behavior among patients according to several covariates.

Results. The mean age at diagnostic time was found to be $55.5(16.1-82.4)$ years with a mean survival of 39.2 $(3.5-100.1 ; \mathrm{SE}=2.6)$ months. Across FIGO stage I, II, III, and IV patients had a mean CA-125 AUC of 18.2, 24.6, 147.8, and 574.6 IU/ml*days, respectively $(P<$ $0.05)$. At the evaluation date, living patients had a mean CA-125 AUC of 40.1 in contrast to $234.1 \mathrm{IU} / \mathrm{m}{ }^{*}$ days $(P<0.05)$ for deceased ones. Patients with a complete response to primary chemotherapy had a mean CA-125 AUC of 48.8, while patients with a partial response had a mean of $251.7 \mathrm{IU} / \mathrm{ml}^{*}$ days, and patients with no response or disease progression had a mean of $316.5 \mathrm{IU} / \mathrm{ml}$ days $(P<0.05)$. The best CA125 AUC performance is in predicting patient complete response to chemotherapy with a cut-off of $100 \mathrm{IU} / \mathrm{ml}$ days and an accuracy of $82 \%$.

Conclusions. Despite CA-125 AUC high correlation with the FIGO stage, residual disease, and patient final outcome, the main interest of CA-125 AUC calculation is to evaluate the treatment efficacy and to foresee a full chemotherapy response. Further studies should be carried out before extrapolating these results to other data sets.
\end{abstract}

(C) 2005 Elsevier Inc. All rights reserved.

Keywords: CA-125 kinetics; Ovarian cancer; Prognostic factors

\section{Introduction}

Ovarian cancer, generally treated with combination first line chemotherapy after cytoreduction surgery $[1,2]$, has the highest mortality rate of all invasive cancers of the gynecological system. Bast et al. [3] first described a radioimmunoassay that could detect CA-125 (Cancer Antigen 125) in the serum of ovarian cancer patients [3]. CA-125 serum concentration is usually adopted to evaluate the clinical situation in ovarian cancer patients and the rate of decline in CA-125 during primary chemotherapy has

\footnotetext{
* Corresponding author. Fax: +351 239855099.

E-mail address: acfalcao@ff.uc.pt (A. Falcão).
}

been an important prognostic factor in several multivariate analyses [4]. The postoperative serum CA-125 level is an independent prognostic factor in patients with invasive ovarian cancer [5], and CA-125 tumor marker half-life $\left(t_{1 / 2}\right)$ and tumor marker doubling time (DT) are often used as kinetic parameters for the evaluation of clinical response and follow-up of patients with ovarian cancer [6]. Serum CA-125 half-life during early chemotherapy is an independent prognostic factor for both the achievement of a pathologically complete response and the survival of patients with advanced epithelial ovarian cancer [7], and several studies report that the greatest difference in progression rate was found at a $t_{1 / 2}$ of 20 days [8-11]. Nevertheless, although CA-125 level before the 3rd course of chemotherapy was considered the best prognostic 
indicator by Fayers et al., it was classified inaccurately for clinical use [12].

In addition to CA-125 kinetic parameters, several other prognostic factors can be used in the management of ovarian cancer: the FIGO (Fédération Internationale de Gynécologie et d'Obstrétrique) tumor stage, tumor grade, tumor biology, overexpression of the HER-2/neu oncogene, residual disease after initial cytoreductive surgery, and rate of response to chemotherapy [13].

CA-125 tumor marker kinetics are more important than the isolated value of CA-125 serum concentration for patient prognosis, and in the present work, we propose a new kinetic parameter: the CA-125 area under the curve (AUC) and its relation with the FIGO stage, patient final state, tumor grade, residual disease, and primary chemotherapy response. Additionally, the influence of this new prognostic factor to overall survival in patients with ovarian cancer was also studied in our population.

\section{Patients and methods}

Retrospective clinical information was gathered from 339 patients with a diagnosis of ovarian cancer at the Gynaecology Service of Coimbra University Hospital (CUH) main database from 1990 to 2000. In addition, CA-125 serum levels of these patients were obtained from the Pathology Service (Hormonology and Drug Monitoring Laboratory) of CUH. Only 92 patients were included in the present analysis due to the restriction of our inclusion criteria: patients that underwent primary line chemotherapy within 4 months after submission to cytoreductive surgery

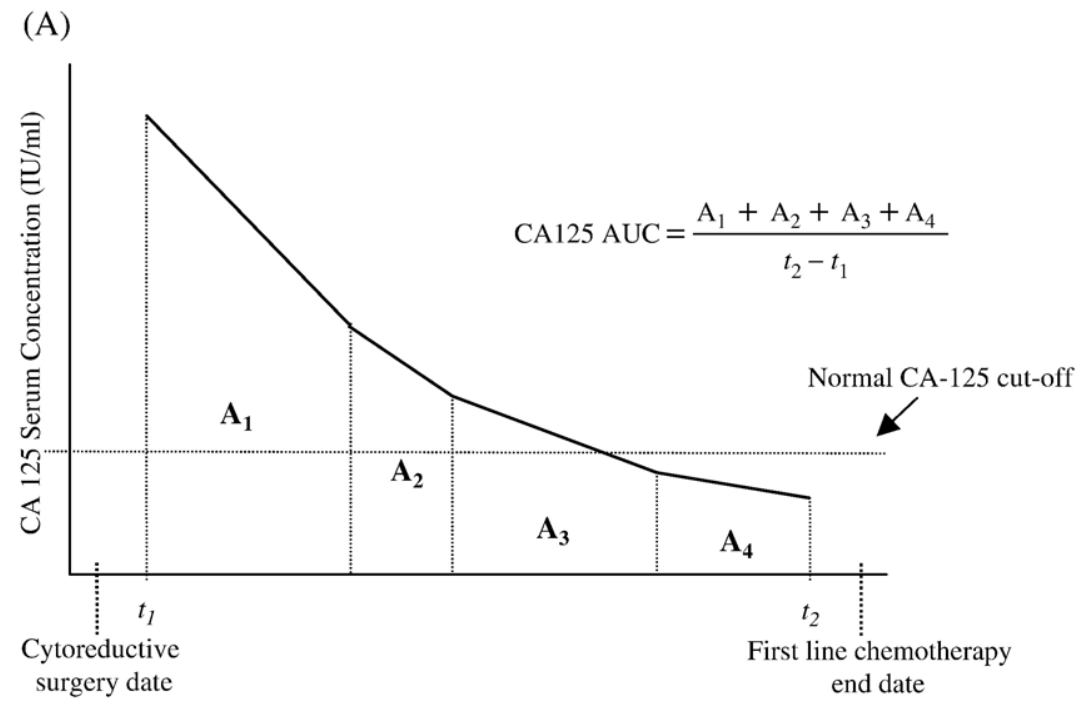

(B)

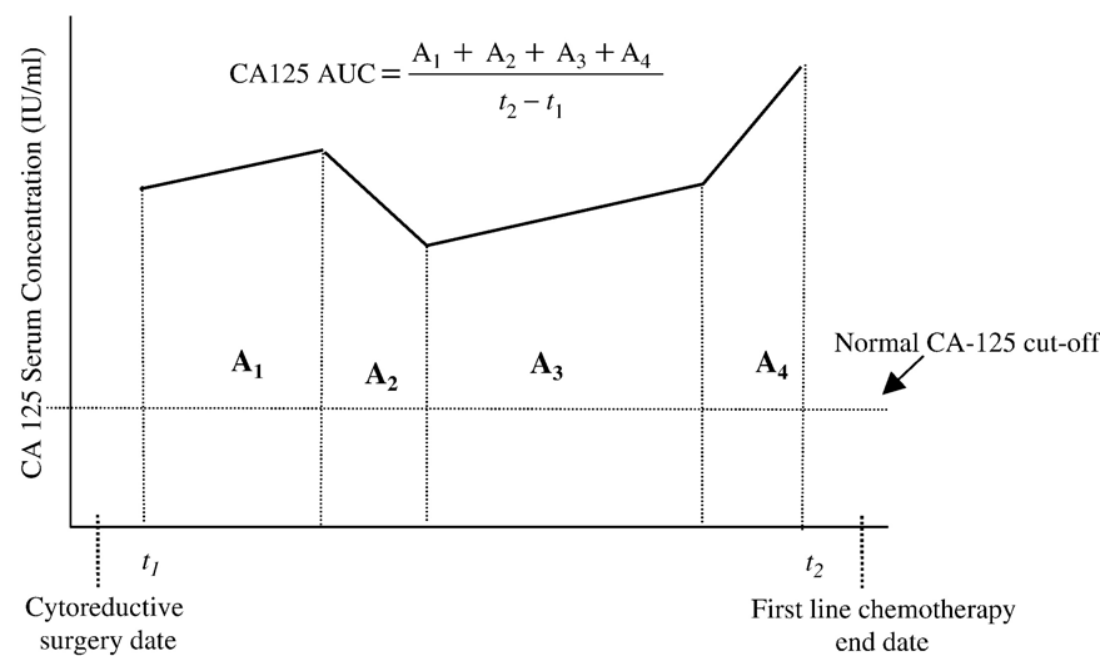

Fig. 1. CA-125 AUC hypothetical determination in a patient with ovarian cancer treated with first line chemotherapy after cytoreductive surgery; (A) patient with an optimal CA-125 serum levels evolution during treatment; (B) patient with an unstable CA-125 serum levels during treatment. 
and with a minimum of three CA-125 serum concentrations between the time of surgery and the end of chemotherapy. For each patient, CA-125 AUC was calculated using the following formula:

$\mathrm{CA}-125 \mathrm{AUC}=\left(\right.$ sum of all trapezoid are as between $C_{1}$ and $\left.C_{2}\right) /\left(t_{2}-t_{1}\right)$

where $C_{1}$ is the first CA-125 serum concentration after the cytoreduction surgery and $C_{2}$ is the last CA-125 serum concentration before the end of first line chemotherapy, and $t_{1}$ and $t_{2}$ are the corresponding dates for $C_{1}$ and $C_{2}$, respectively (Fig. 1). A statistical analysis was conducted. The Mann-Whitney $U$ test (for two groups) and KruskalWallis test (for three or more groups) were used to compare the CA-125 AUC across subgroups of patients, depending on the FIGO stage, patient final state, tumor grade, histological type, residual disease $(>2 \mathrm{~cm})$, and response to primary chemotherapy. The area under the Receiver Operating Characteristic (ROC) curve was established for the discrimination by CA-125 AUC in predicting the patient final state, overall survival equal or superior to 1,3 , and 5 years, and a full response to chemotherapy. Sensitivity, specificity, positive predictive values (PPV), and overall accuracy were also determined for several CA-125 AUC cutoffs. In addition, a multivariant regression analysis based on the Cox proportional hazard model to test the variation of CA-125 AUC in relation to the overall survival time was also carried out. A $P$ level $\leq 0.05$ was considered significant.

\section{Results}

The mean age at diagnostic time was found to be 55.5 (16.1-82.4; $\mathrm{SE}=1.65)$ years with a mean overall survival of 39.2 (3.5-100.1; SE = 2.6) months. According to the FIGO tumor stage, nineteen $(20.7 \%)$ patients had stage I, eleven $(11.9 \%)$ had stage II, fifty-one $(55.4 \%)$ had stage III, nine $(9.8 \%)$ had stage IV, and in two $(2.2 \%)$ patients this information was missing. Eighty-two (89.1\%) patients had epithelial ovarian cancer for histological type. Twelve $(13.0 \%)$ patients had a tumor grade 1, twenty-nine $(31.5 \%)$ a tumor grade 2 , nine $(9.8 \%)$ had a tumor grade 3 , and forty-two $(45.7 \%)$ patients had no tumor grade information. Thirty-six (39.1\%) patients had a residual tumor greater than $2 \mathrm{~cm}$ after surgery. The mean duration of primary chemotherapy was $4.1(0.7-10.2$; $\mathrm{SE}=0.15)$ months: fifty-two $(56.5 \%)$ patients had a complete response to primary chemotherapy, twenty-two $(23.9 \%)$ had a partial response, thirteen $(14.1 \%)$ had no response or a disease progression, and five $(5.4 \%)$ patients had missing information for the response to chemotherapy. At evaluation date, fifty $(54.3 \%)$ patients were deceased while forty-two $(45.7 \%)$ were alive. Eighty patients $(86.9 \%)$ had at least 1 year survival, forty-four $(47.8 \%)$ had at least 3 years while only twenty-one $(22.8 \%)$ had more than 5 years survival (Table 1).
Table 1

Summary of patient characteristics

Patient count $(\%)$

FIGO stage

I $19(20.7)$

II $11(11.9)$

III $51(55.4)$

IV $9(9.8)$

Missing $2(2.2)$

Patient final state

Deceased $\quad 50(54.3)$

Alive $\quad 42(45.7)$

Tumor grade

$1212(13.0)$

$229(31.5)$

$3 \quad 9(9.8)$

Missing 42 (45.7)

Histological type

Epithelial $\quad 82(89.1)$

Other $\quad 8(8.7)$

Missing $2(2.2)$

Residual disease $(>2 \mathrm{~cm})$

Yes $\quad 36(39.1)$

No $\quad 53$ (57.6)

Missing 3 (3.3)

Primary chemotherapy response

Complete response (CR) 52 (56.5)

Partial response (PR) $22(23.9)$

Without response or disease progression (WR/DP) 13 (14.1)

Missing

$5(5.4)$

Overall survival

$\geq 1$ year $\quad 80(86.9)$

$\geq 3$ years $44(47.8)$

$\geq 5$ years $21(22.8)$

The CA-125 AUC across groups of patients is shown in Table 2. Patients with FIGO stage I, II, III, and IV had a mean CA-125 AUC of $18.2(\mathrm{SE}=2.4) \mathrm{IU} / \mathrm{ml}^{*}$ days, 24.6 $(\mathrm{SE}=7.6) \mathrm{IU} / \mathrm{ml}^{*}$ days, $147.8(\mathrm{SE}=30.8) \mathrm{IU} / \mathrm{ml}^{*}$ days, and $574.6(\mathrm{SE}=134.6) \mathrm{IU} / \mathrm{ml} *$ days, respectively $(P<0.05)$ (Fig. 2). Patients with a tumor grade 1, 2, and 3 had a mean CA-125 AUC of 100.1 (45.5) IU/ml*days, 158.1 (43.0) IU/ $\mathrm{ml}^{*}$ days, and $238.8(114.0) \mathrm{IU} / \mathrm{ml}^{*}$ days, respectively $(P>$ $0.05)$. Patients with residual disease $(>2 \mathrm{~cm})$ had a mean CA-125 AUC of $207.4(\mathrm{SE}=48.3) \mathrm{IU} / \mathrm{ml} *$ days, while patients without residual disease had $97.9(\mathrm{SE}=30.0) \mathrm{IU} /$ $\mathrm{ml}^{*}$ days $(P<0.05)$. At the evaluation date, living patients had a mean CA-125 AUC of $40.1(\mathrm{SE}=10.5) \mathrm{IU} / \mathrm{ml}$ *days in contrast to deceased patients, who had a CA-125 AUC of $234.1(\mathrm{SE}=44.4) \mathrm{IU} / \mathrm{ml}$ *days $(P<0.05)$. Fifty-two patients had a complete response to primary chemotherapy with a mean CA-125 AUC of $48.8 \mathrm{IU} / \mathrm{ml}^{*}$ days ( $\mathrm{SE}=15.9$ ) while twenty-two patients had a partial response and thirteen patients had no response or disease progression with a mean CA-125 AUC of $251.7 \mathrm{IU} / \mathrm{ml}^{*}$ days $(\mathrm{SE}=65.8)$ and a mean 
Table 2

CA-125 AUC behavior among patients according to several covariates

\begin{tabular}{|c|c|c|c|}
\hline Group & $\begin{array}{l}\text { Mean CA-125 } \\
\text { AUC } \\
\text { (standard error) } \\
{\left[\mathrm{IU} / \mathrm{ml}^{*} \text { days }\right]}\end{array}$ & $\begin{array}{l}\text { Median CA-125 } \\
\text { AUC }\left(Q_{25}-Q_{75}\right) \\
{\left[\mathrm{IU} / \mathrm{ml}^{*} \text { days }\right]}\end{array}$ & $P$ value \\
\hline \multicolumn{4}{|l|}{ FIGO stage } \\
\hline I & $18.2(2.4)$ & $14.6(12.0-20.1)$ & $P<0.05$ \\
\hline II & $24.6(7.6)$ & $14.4(9.8-20.5)$ & \\
\hline III & $147.8(30.8)$ & $54.2(23.5-199.5)$ & \\
\hline IV & $574.6(134.6)$ & $676.3(118.6-973.3)$ & \\
\hline \multicolumn{4}{|l|}{ Patient final state } \\
\hline Deceased & $234.1(44.4)$ & $83.2(23.5-319.5)$ & $P<0.05$ \\
\hline Alive & $40.1(10.5)$ & $16.5(12.0-34.0)$ & \\
\hline \multicolumn{4}{|l|}{ Tumor grade } \\
\hline 1 & $100.1(45.5)$ & $19.8(12.3-146.9)$ & NS \\
\hline 2 & $158.1(43.0)$ & $65.8(16.6-230.7)$ & \\
\hline 3 & $238.8(114.0)$ & $44.8(37.3-375.2)$ & \\
\hline \multicolumn{4}{|l|}{ Residual disease $(>2 \mathrm{~cm})$} \\
\hline Yes & $207.4(48.3)$ & $98.1(31.3-237.9)$ & $P<0.05$ \\
\hline No & $97.9(30.0)$ & $16.6(12.0-44.8)$ & \\
\hline \multicolumn{4}{|c|}{ Primary chemotherapy response } \\
\hline Complete response (CR) & $48.8(15.9)$ & $16.8(12.0-34.1)$ & $P<0.05$ \\
\hline Partial response (PR) & $251.7(65.8)$ & $98.1(51.9-319.5)$ & \\
\hline $\begin{array}{l}\text { Without response or } \\
\text { disease progression } \\
\text { (WR/DP) }\end{array}$ & $316.5(107.5)$ & $116.8(54.2-344.3)$ & \\
\hline
\end{tabular}

of $316.5 \mathrm{IU} / \mathrm{ml}^{*}$ days $(\mathrm{SE}=107.5)$, respectively $(P<0.05)$ (Fig. 3).

For predicting the patient final state, the best accuracy (74\%) was achieved at CA-125 AUC $\leq 100 \mathrm{IU} / \mathrm{ml}$ *days (ROC AUC $=0.77$ ). To predict patient survival, the most accurate was $87 \%$ (CA-125 AUC $\leq 1000 \mathrm{IU} / \mathrm{ml}$ days), $72 \%$ (CA-125 AUC $\leq 100 \mathrm{IU} / \mathrm{ml}$ *days), and 66\% (CA-125 AUC $\leq 25 \mathrm{IU} / \mathrm{ml}^{*}$ days) for a 1-, 3-, and 5-year overall survival, respectively (ROC AUC $=0.67,0.75$, and 0.73 ). In predicting a complete response to chemotherapy (ROC AUC $=0.87$ ), the best CA-125 AUC cut-off was $100 \mathrm{IU} /$

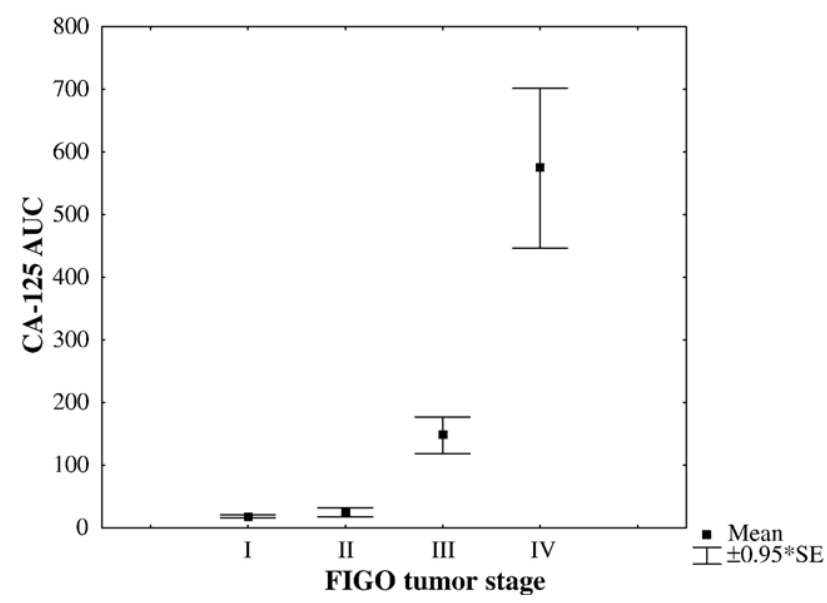

Fig. 2. CA-125 mean plot according to patients' FIGO tumor stage.

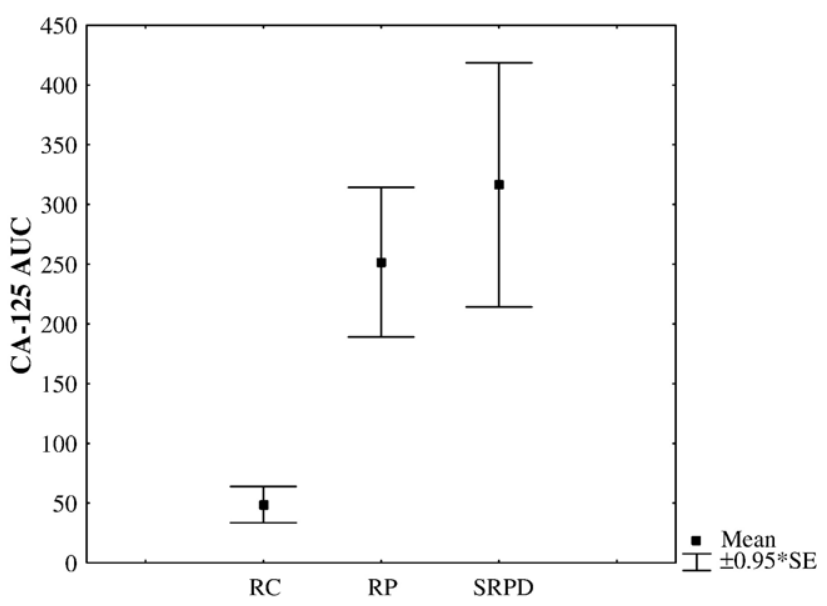

Fig. 3. CA-125 mean plot according to patient response to primary chemotherapy. $(\mathrm{CR}=$ complete response; $\mathrm{PR}=$ partial response; $\mathrm{WRDP}=$ without response or disease progression.)

$\mathrm{ml}$ *days with an accuracy of $82 \%$ (Table 3 ). We estimated the parameters in the Cox proportional hazard model using overall survival as a dependent variable, CA-125 AUC as an independent variable, and the patient final state ("Alive" or "Deceased") as a censoring variable. FIGO tumor stage, residual disease, and primary chemotherapy response were stratification variables (Table 4). Fig. 4 shows the survival functions for different values of CA-125 AUC produced with the Cox model without any stratification variable.

\section{Discussion}

Many authors have studied the CA-125 kinetic in monitoring the ovarian cancer patient. Kinetic parameters prove to be more useful than rough serum concentration alone. Van der Burg et al. [8], Hawkins et al. [9], Verda et al. [10], S. Ėolakoviæ et al. [11], and others used the CA-125 half-life value to evaluate patient survival, finding a $t_{1 / 2}$ of 20 days to be a breakpoint between a "good" and "poor" prognosis.

Buller et al. showed that the rate of decline of CA-125 in effectively treated ovarian cancer is described by an exponential model and his study suggests that it is possible to predict overall survival, which patients have residual disease at reassessment laparotomy, who will be free of disease, and who will have a recurrence $[14,15]$.

In a review article, Jean-Michel et al. [6] accentuated the value of different serum marker kinetic parameters in the monitoring of patients in several types of cancer.

To increase the value of CA-125 kinetics as a prognostic factor in ovarian cancer, we proposed and studied a new kinetic parameter: CA-125 AUC. As can be seen in Figs. $1 \mathrm{~A} / \mathrm{B}$, the CA-125 AUC calculation is independent of the shape presented by the CA-125 serum concentrations, making CA-125 AUC a more suitable kinetic parameter than CA-125 half-life (Fig. 1A). In addition, the CA-125 
Table 3

Sensitivity, specificity, positive predictive value (PPV), and accuracy of several CA-125 AUC cut-offs for predicting the patient final state, overall survival, and chemotherapy complete response $(\mathrm{SE}=$ standard error)

\begin{tabular}{|c|c|c|c|c|c|c|c|c|c|c|c|}
\hline CA-125 AUC [IU/ml*days] & $\leq 25$ & $\leq 50$ & $\leq 100$ & $\leq 200$ & $\leq 300$ & $\leq 400$ & $\leq 500$ & $\leq 600$ & $\leq 800$ & $\leq 1000$ & ROC AUC (SE) \\
\hline \multicolumn{12}{|c|}{ CA-125 cut-off to predict patient final state "alive" } \\
\hline Sensitivity & 0.62 & 0.86 & 0.86 & 0.95 & 0.97 & 1.00 & 1.00 & 1.00 & 1.00 & 1.00 & \multirow[t]{4}{*}{$0.77(0.05)$} \\
\hline Specificity & 0.72 & 0.64 & 0.64 & 0.32 & 0.30 & 0.20 & 0.20 & 0.14 & 0.08 & 0.04 & \\
\hline PPV & 0.65 & 0.67 & 0.67 & 0.54 & 0.54 & 0.51 & 0.51 & 0.50 & 0.48 & 0.47 & \\
\hline Accuracy & 0.67 & 0.74 & 0.74 & 0.61 & 0.61 & 0.57 & 0.57 & 0.53 & 0.50 & 0.48 & \\
\hline \multicolumn{12}{|c|}{ CA-125 cut-off to predict patient overall survival $\geq 1$ year } \\
\hline Sensitivity & 0.48 & 0.63 & 0.63 & 0.81 & 0.84 & 0.90 & 0.90 & 0.94 & 0.98 & 0.99 & \multirow[t]{4}{*}{$0.67(0.08)$} \\
\hline Specificity & 0.83 & 0.67 & 0.67 & 0.25 & 0.25 & 0.17 & 0.17 & 0.17 & 0.17 & 0.08 & \\
\hline PPV & 0.95 & 0.93 & 0.93 & 0.88 & 0.88 & 0.88 & 0.88 & 0.88 & 0.89 & 0.88 & \\
\hline Accuracy & 0.52 & 0.63 & 0.63 & 0.74 & 0.76 & 0.80 & 0.80 & 0.84 & 0.87 & 0.87 & \\
\hline \multicolumn{12}{|c|}{ CA-125 cut-off to predict patient overall survival $\geq 3$ years } \\
\hline Sensitivity & 0.64 & 0.82 & 0.82 & 0.98 & 0.98 & 0.98 & 0.98 & 0.98 & 0.98 & 1.00 & \multirow[t]{4}{*}{$0.75(0.05)$} \\
\hline Specificity & 0.75 & 0.63 & 0.63 & 0.35 & 0.31 & 0.19 & 0.19 & 0.13 & 0.06 & 0.04 & \\
\hline PPV & 0.70 & 0.67 & 0.67 & 0.58 & 0.57 & 0.52 & 0.52 & 0.51 & 0.49 & 0.49 & \\
\hline Accuracy & 0.70 & 0.72 & 0.72 & 0.65 & 0.63 & 0.57 & 0.57 & 0.53 & 0.50 & 0.50 & \\
\hline \multicolumn{12}{|c|}{ CA-125 cut-off to predict patient overall survival $\geq 5$ years } \\
\hline Sensitivity & 0.71 & 0.90 & 0.90 & 1.00 & 1.00 & 1.00 & 1.00 & 1.00 & 1.00 & 1.00 & \multirow[t]{4}{*}{$0.73(0.06)$} \\
\hline Specificity & 0.65 & 0.51 & 0.51 & 0.25 & 0.23 & 0.14 & 0.14 & 0.10 & 0.08 & 0.04 & \\
\hline PPV & 0.38 & 0.35 & 0.35 & 0.28 & 0.28 & 0.26 & 0.26 & 0.25 & 0.44 & 0.43 & \\
\hline Accuracy & 0.66 & 0.60 & 0.60 & 0.42 & 0.40 & 0.34 & 0.34 & 0.30 & 0.47 & 0.45 & \\
\hline \multicolumn{12}{|c|}{ CA-125 cut-off to predict patient complete response to chemotherapy } \\
\hline Sensitivity & 0.65 & 0.85 & 0.85 & 0.94 & 0.96 & 0.98 & 0.98 & 0.98 & 1.00 & 1.00 & \multirow[t]{4}{*}{$0.87(0.04)$} \\
\hline Specificity & 0.89 & 0.77 & 0.77 & 0.37 & 0.34 & 0.23 & 0.23 & 0.14 & 0.11 & 0.06 & \\
\hline PPV & 0.89 & 0.85 & 0.85 & 0.69 & 0.68 & 0.65 & 0.65 & 0.63 & 0.63 & 0.61 & \\
\hline Accuracy & 0.75 & 0.82 & 0.82 & 0.71 & 0.71 & 0.68 & 0.68 & 0.64 & 0.64 & 0.62 & \\
\hline
\end{tabular}

AUC is less disturbed by peak phenomena (especially after surgery) and sources of variability (i.e., intra-subject and assay variability).

In the present work, the poor correlation between CA$125 \mathrm{AUC}$ and tumor grade is perhaps the consequence of the high number of patients without this information. Inversely, CA-125 AUC is highly correlated with the FIGO stage in which lower values are related with stage I and II, middle values with stage III, and higher values with stage IV. Patients with stage IV have a mean CA-125 AUC 31.6 times greater than patients with stage I. CA-125 AUC is also correlated with residual disease for patients with a residual tumor $>2 \mathrm{~cm}$ after initial cytoreductive surgery (mean CA125 AUC 2.1 times greater). CA-125 AUC is also correlated with patient final state in deceased patients, having a mean

Table 4

Cox proportional hazard model results for CA-125 AUC as an independent factor for predicting patient overall survival

\begin{tabular}{lcl}
\hline $\begin{array}{l}\text { Dependent: overall survival; independent: CA-125 AUC; censoring } \\
\text { variable: patient final state (deceased/alive) }\end{array}$ \\
\hline Stratified by: & Chi-square & $P$ value \\
\hline None & 18.19 & $P<0.05$ \\
FIGO tumor stage (I, II, III, IV) & 6.42 & $P<0.05$ \\
Residual disease ( $>2 \mathrm{~cm})$ & 14.80 & $P<0.05$ \\
Primary chemotherapy response & 1.83 & $\mathrm{NS}$ \\
$\quad(\mathrm{CR}$, PR, WR/DP) & &
\end{tabular}

CA-125 AUC 5.8 times greater than living. As shown by the Cox proportional hazard model, the CA-125 AUC is an independent prognostic factor for patient overall survival and patients with a lower CA-125 AUC have a better overall survival than patients with a higher CA-125 AUC.

Concerning survival forecast, the best CA-125 AUC cutoff was $100 \mathrm{IU} / \mathrm{ml}^{*}$ days, obtained for predicting an overall survival $\geq 3$ years, the patient final state "alive", and the complete response to chemotherapy, with an accuracy of $72 \%, 74 \%$, and $82 \%$, respectively. In fact, it seems clear that

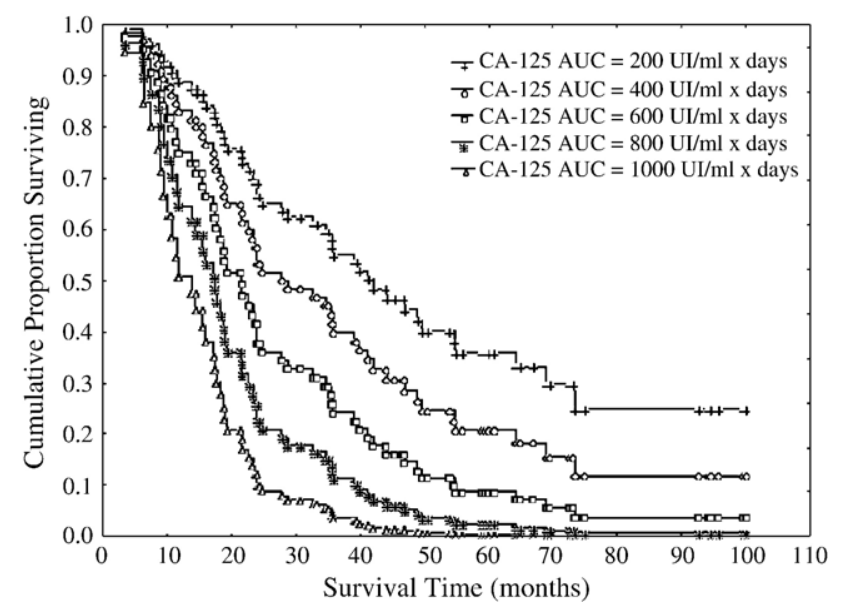

Fig. 4. Survival function for hypothetical CA-125 AUC values. 
lower CA-125 AUC values are associated with a complete response, while higher values are associated with a partial response or even a disease progression. Therefore, CA-125 AUC could be a useful measure of the primary treatment efficacy, not only to evaluate the cytoreductive surgery but also the chemotherapy cocktail adopted. Regarding the CA125 AUC kinetic, the objective of initial treatment (cytoreductive surgery and primary chemotherapy) of ovarian cancer is to produce the lowest CA-125 AUC possible. In addition, the CA-125 AUC kinetic parameter could be useful as an end-point in the development of new chemotherapy drugs or to establish new guidelines for the primary treatment of ovarian cancer.

Finally, CA-125 AUC presents some benefits over other kinetic parameters: it is easier to calculate and modelindependent. Nevertheless, further studies should be carried out in order to compare CA-125 AUC with other prognostic factors used in the management of ovarian cancer patients and caution should be exercised before extrapolation of the present results to different data sets.

\section{References}

[1] Vicki VB. Treatment options for ovarian cancer. Clin Obstet Gynecol 2001;44(3):522-30.

[2] Eduard EP, Mack NB. Epithelial ovarian cancer: prevention, diagnosis, and treatment. CA Cancer J Clin 1999;49:297-320.

[3] Bast Jr RC, Klug TL, St. John E, Jenison E, Niloff JM, Lazarus H. A radioimmunoassay using a monoclonal antibody to monitor the course of epithelial ovarian cancer. N Engl J Med 1983;309:883-7.

[4] Bast Jr RC, Xu FJ, Yu YH, Barnhill S, Zhang Z, Mills GB. CA 125: the past and the future. Int J Biol Markers 1998;13(4):179-87.

[5] Amin PHM, Gunnar BK, Janne K, Ole PB, Vera MA, Claes GT. Prognostic value of pre- and postoperative serum CA 125 levels in ovarian cancer: new aspects and multivariate analysis. Obstet Gynecol 1992;79:1002-10.

[6] Jean-Michel B, François T, Christine A, Jacqueline C, Alain D, Nelly $\mathrm{J}$, et al. Kinetic of serum marker concentrations and usefulness in clinical monitoring. Clin Chem 1999;45(10):1695-707.

[7] Angiolo G, Paolo Z, Fabio L, Tiziano M, Enrico S, Tiziana B, et al. Serum half-life of CA 125 during early chemotherapy as an independent prognostic variable for patients with advanced epithelial ovarian cancer: results of a multicentric Italian study. Gynecol Oncol 1995;58:42-7.

[8] Van Der Burg MEL, Lammes FB, Van Putten WLJ, Stoter G. Ovarian cancer: the prognostic value of the serum half-life of $\mathrm{CA}$ 125 during induction chemotherapy. Gynecol Oncol 1988; 30:307-12.

[9] Hawkins RE, Roberts K, Wiltshaw E, Mundy J, Fryatt IJ, McCready VR. The prognostic significance of the half-life of serum CA 125 in patients responding to chemotherapy for epithelial ovarian carcinoma. Br J Obstet Gynaecol 1989;96:1395-9.

[10] Verda JH, Lee D, Michael H, John TS, Andrew B, Daniel LCP, Bast Jr RC. The prognostic significance of CA 125 half-life in patients with ovarian cancer who have received chemotherapy after surgical cytoreduction. Am J Obstet Gynecol 1990;163: 1164-7.

[11] Čolakovič S, Lukiç V, Mitroviç L, Jeliç S, Šušnjar S, Marinkoviç J. Prognostic value of CA 125 kinetics and half-life in advanced ovarian cancer. Int J Biol Markers 2000;15(2):147-52.

[12] Fayers PM, Rusting G, Wood R, Nelstrop A, Leonard RCF, Wilkinson $\mathrm{P}$, et al. The prognostic value of serum CA 125 in patients with advanced ovarian carcinoma: an analysis of 573 patients by the medical research council working party on gynaecological cancer. Int J Gynecol Cancer 1993;3:285-92.

[13] Christine HH, Jonathan SB. Ovarian cancer: epidemiology, biology, and prognostic factors. Semin Surg Oncol 2000;19:3-10.

[14] Richard EB, Michael LB, Jeffrey DB, Alberto M, Philip JDS. CA 125 regression: a model for epithelial ovarian cancer response. Am J Obstet Gynecol 1991;165:360-7.

[15] Richard EB, Michael LB, Jeffery DB, Alberto M, Philip JDS Serum CA 125 regression in epithelial ovarian cancer: correlation with reassessment findings and survival. Gynecol Oncol 1992;47: $87-92$. 